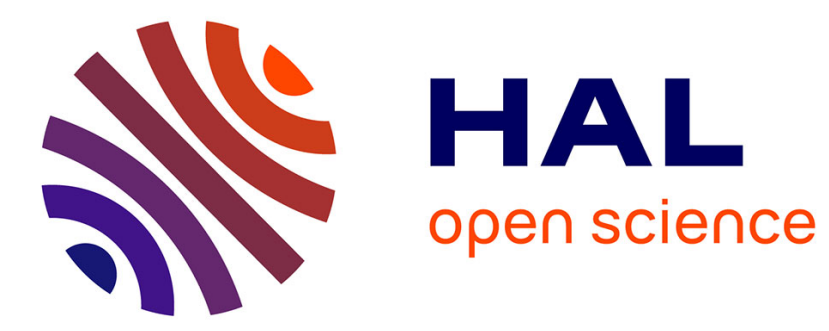

\title{
Local Frederiks transitions near a solid/nematic interface
}

E. Dubois-Violette, P.-G. de Gennes

\section{To cite this version:}

E. Dubois-Violette, P.-G. de Gennes. Local Frederiks transitions near a solid/nematic interface. Journal de Physique Lettres, 1975, 36 (10), pp.255-258. 10.1051/jphyslet:019750036010025500 . jpa00231202

\section{HAL Id: jpa-00231202 https://hal.science/jpa-00231202}

Submitted on 1 Jan 1975

HAL is a multi-disciplinary open access archive for the deposit and dissemination of scientific research documents, whether they are published or not. The documents may come from teaching and research institutions in France or abroad, or from public or private research centers.
L'archive ouverte pluridisciplinaire HAL, est destinée au dépôt et à la diffusion de documents scientifiques de niveau recherche, publiés ou non, émanant des établissements d'enseignement et de recherche français ou étrangers, des laboratoires publics ou privés. 


\title{
LOCAL FREDERIKS TRANSITIONS NEAR A SOLID/NEMATIC INTERFACE
}

\author{
E. DUBOIS-VIOLETTE \\ Physique des Solides, Université Paris-Sud, 91405 Orsay, France \\ P. G. DE GENNES \\ Physique de la Matière Condensée, Collège de France, 75231 Paris Cedex 05, France
}

(Reçu le 9 juin 1975, accepté le 9 juillet 1975)

\begin{abstract}
Résumé. - On considère un interface solide-nématique près duquel les couples de van der Waals favorisent un type d'alignement (par exemple homéotrope) alors que les interactions stériques au contact tendent à donner un autre type (par exemple planaire). En faisant varier le rapport des deux forces impliquées, on peut rencontrer deux transitions : planaire $\leftrightarrow$ conique $\leftrightarrow$ homéotrope. Ceci rappelle certaines observations récentes de Ryschenkow sur des surfaces de verre recouvertes de noir de fumée.
\end{abstract}

\begin{abstract}
We consider a solid/nematic interface where long range van der Waals torques favor homeotropic alignment, while a direct steric effect tends to induce a planar alignment. Depending on the relative strength of the two contributions, one may find two transitions : planar $\leftrightarrow$ conical and conical $\leftrightarrow$ homeotropic. This is reminiscent of recent observations by Ryschenkow and Kléman on glass surfaces coated with carbon black. Similar local Frederiks transitions could occur by competition between two distinct planar arrangments.
\end{abstract}

Nematic and cholesteric fluids are rather sensitive to long range van der Waals forces [1-4]. In the present note, we want to discuss one particular situation near a solid/nematic interface $(z=d)$, where weak short range anchoring forces favor one alignment $(\theta=\pi / 2$ on figure 1$)$ while long range torques favor the opposite $(\theta=0)$. We shall show that in such a case, a remarkable local Frederiks transition [5] may occur. The energy is (per $\mathrm{cm}^{2}$ of interface)

$$
\begin{aligned}
F=- & \frac{1}{2} A \sin ^{2} \theta_{0}+ \\
& +\int_{d}^{\infty}\left[\frac{1}{2} U(z) \sin ^{2} \theta+\frac{1}{2} K\left(\frac{\mathrm{d} \theta}{\mathrm{d} z}\right)^{2}\right] \mathrm{d} z .
\end{aligned}
$$

Here $A$ describes the short range anchoring, and is assumed positive. $\theta_{0}=\theta(d)$ is the angle at the interface. $U(z)$ describes the van der Waals torques. If the solid substrate (separated from the nematic by a small passive gap of thickness $d$ ) is uniaxial, $U(z)$ contains a leading contribution proportional to $z^{-3}$ [1]. If the substrate is isotropic, the decrease is faster $\left(z^{-5}\right) . K$ is an average elastic constant [5]. We assume for simplicity that the conditions for $z \rightarrow \infty$

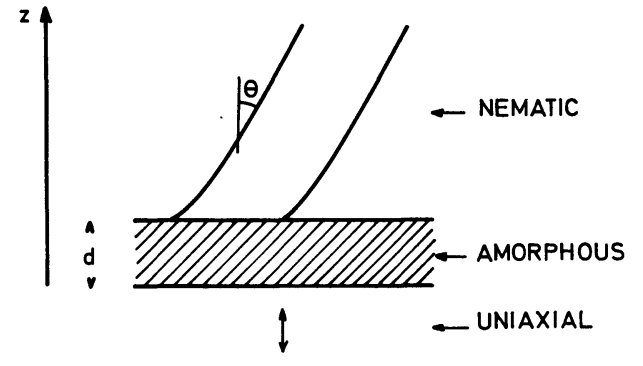

FIG. 1. - One example of competition between long range van der Waals torques (favoring $\theta=0$ ) and surface anchoring (favoring $\theta=\pi / 2$ ).

correspond to zero torque $(\mathrm{d} \theta / \mathrm{d} z \rightarrow 0)$ [6]. The equilibrium equation is :

$$
\frac{\mathrm{d}^{2} \theta}{\mathrm{d} z^{2}}=\frac{U(z)}{K} \sin \theta \cos \theta
$$

together with the torque balance at the intertace

$$
\left.K \frac{\mathrm{d} \theta}{\mathrm{d} z}\right|_{d}=-A \sin \theta_{0} \cos \theta_{0} .
$$

We shall focus our discussion on the two uniform states $\theta=0$ and $\theta=\pi / 2$, and on this local stability. 
1. The energy difference $F_{0}-F_{\pi / 2}$ vanishes when

$$
A=A_{\mathrm{c}} \equiv \int_{d}^{\infty} U(z) \mathrm{d} z
$$

If the anchoring energy $A$ is due to grooves on the surface [7] [5] it is proportional to $K$ and thus more or less to $S^{2}$ (where $S$ is the order parameter) while $U$ is roughly linear in $S$. Thus temperature variations may allow to cross the threshold [4]. Eq. (4) would correspond to a first order transition. But more gradual transitions can occur, as we shall see.

2. Let us investigate the local stability of the low $A$ $(\theta=0)$ phase. For small $\theta$ the linearized form of eq. (2)

$$
\frac{\mathrm{d}^{2} \theta}{\mathrm{d} z^{2}}=\frac{U(z)}{K} \theta
$$

has a solution with upward curvature shown in figure $2 a$. The torque at the interface may be transformed into

$$
-\left.K \frac{\mathrm{d} \theta}{\mathrm{d} z}\right|_{d}=\int_{d}^{\infty} U(z) \theta(z) \mathrm{d} z
$$

Eq. (3) shows that instability sets in when this equal to $A \theta_{0}$ : Thus, we find another threshold

$$
A^{\prime}=\int_{d}^{\infty} U(z) \frac{\theta(z)}{\theta(d)} \mathrm{d} z
$$

From figure $2 a$ (or from eq. (2')) we see that $\theta(z)<\theta(d)$ and $A^{\prime}<A_{\mathrm{c}}$.

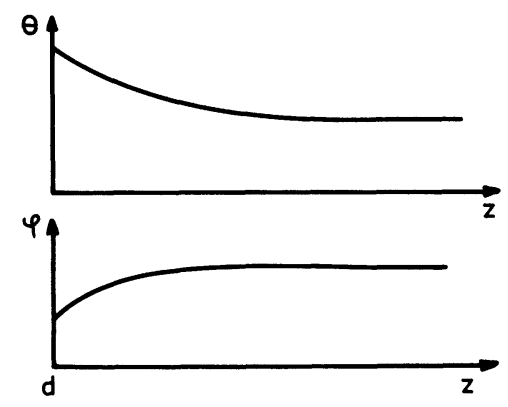

FIG. 2. - Qualitative form of the self consistent distortion near the two thresholds. $a$ ) plot of $\theta$ in the case of small $\theta, b$ ) plot of $\varphi=\pi / 2-\theta$ in the case of small $\varphi$ and sample thickness $d>d_{\mathrm{c}}$.

3. The local stability of the high $A(\theta=\pi / 2)$ phase is discussed by assuming that $\varphi=\frac{\pi}{2}-\theta$ is small. The linearized equations for $\varphi$ and $\theta$ differ only by the change $U \rightarrow-U$. The solution for $\varphi$ has a downward curvature (Fig. $2 b$ ) and $\varphi(z)>\varphi(d)$. The instability threshold is

$$
A^{\prime \prime}=\int_{d}^{\infty} U(z) \frac{\varphi(z)}{\varphi(d)} \mathrm{d} z>A_{\mathrm{c}} .
$$

Thus there is a finite range of $A$ values $A^{\prime}<A<A^{\prime \prime}$ where partial tilt must occur. We shall now show that the transitions at $A^{\prime}$ and $A^{\prime \prime}$ are of second order. For $A$ slightly above $A^{\prime}$, the energy $F$, expanded to fourth order in $\theta$, is

$$
\begin{aligned}
F= & -\frac{1}{2} A\left(\theta_{0}^{2}-\frac{1}{3} \theta_{0}^{4}\right)+ \\
& +\int_{d}^{\infty} \mathrm{d} z\left[\frac{1}{2} U\left(\theta^{2}-\frac{1}{3} \theta^{4}\right)+\frac{1}{2} K\left(\frac{\mathrm{d} \theta}{\mathrm{d} z}\right)^{2}\right] .
\end{aligned}
$$

We evaluate (8) from a variational principle, using as a trial function the solution $\theta(z)$ of the linear problem. Making use of the following relation

$$
\int_{d}^{\infty}\left(u \theta^{2}+K\left(\frac{\mathrm{d} \theta}{\mathrm{d} z}\right)^{2}\right) \mathrm{d} z=-\left.\frac{1}{2} \theta_{0} \frac{\mathrm{d} \theta}{\mathrm{d} z}\right|_{d}=\frac{1}{2} A^{\prime} \theta_{0}^{2}
$$

the result reads :

$$
\left.\begin{array}{l}
F=\frac{1}{2}\left(A^{\prime}-A\right) \theta_{0}^{2}+\frac{1}{6} \theta_{0}^{4}(A-\tilde{A}) \\
\tilde{A}=\int_{d}^{\infty} U(z)\left(\frac{\theta}{\theta_{0}}\right)^{4} \mathrm{~d} z
\end{array}\right\} .
$$

Since $\theta \leqslant \theta_{0}$ we have $\tilde{A}<A^{\prime}<A$. Thus, the coefficient of $\theta_{0}^{4}$ in $F$ is positive, and this ensures that the optimal $\theta_{0}$ is small when $A$ is close to $A^{\prime}$. A similar argument holds for the transition at $A^{\prime \prime}$.

We have computed the values of $A^{\prime}$ and $A^{\prime \prime}$ for the case of an anisotropic substrate, where $U / K=B / Z$ (for the present problem, all the resulting integrals converge rapidly at large $z$, and thus the inclusion of retardation effects [8] is not required, provided that the gap $d$ is smaller than $\sim 1000 \AA$ ). The solutions of the linear problem can be expressed in terms of Bessel functions [4].

3.1 At the lower threshold $\left(A \rightarrow A^{\prime}\right)$ we have (following the notation of ref. [9]) :

$$
\begin{gathered}
\theta=\theta_{0}\left(\frac{z}{d}\right)^{1 / 2} \frac{I_{1}(t)}{I_{1}\left(t_{0}\right)} \\
t=2(B / z)^{1 / 2}, \quad t_{0}=2(B / d)^{1 / 2}
\end{gathered}
$$

giving

$$
A^{\prime}=\frac{1}{4} \frac{K}{(B d)^{1 / 2}} \int_{0}^{t_{0}} t^{2} \frac{I_{1}(t)}{I_{1}\left(t_{0}\right)} \mathrm{d} t
$$

The form of $\theta / \theta_{0}$ is shown on figure $2 a$.

It is also interesting to discuss the shape of $\theta(z)$ at large $z$, as deduced from eq. (10), for $A$ slightly above $A^{\prime}$ :

$$
\begin{aligned}
\theta & =\theta_{\infty}\left[1+\frac{B}{2 z}+\theta\left(\frac{B}{z}\right)^{2}\right], \quad(z \gg B) \\
\theta_{\infty} & =\theta_{0}\left(1-\frac{B_{v}}{2 d}\right) .
\end{aligned}
$$


Thus the distortion contains an average term $\theta_{\infty}(A)$ plus local corrections restricted to a microscopic region of size $B$ near the interface.

Using the relation [9]

$$
\int_{0}^{t_{0}} t^{2} I_{1}(t) \mathrm{d} t=t_{0}^{2} I_{2}\left(t_{0}\right)
$$

one obtains the dimensionless expression of the anchoring energy threshold

$$
\frac{A^{\prime} B}{K}=\left(\frac{B}{d}\right)^{3 / 2} \frac{I_{2}\left(2 \sqrt{\frac{B}{d}}\right)}{I_{1}\left(2 \sqrt{\frac{B}{d}}\right)} . \quad \text { (see Fig. 3) }
$$

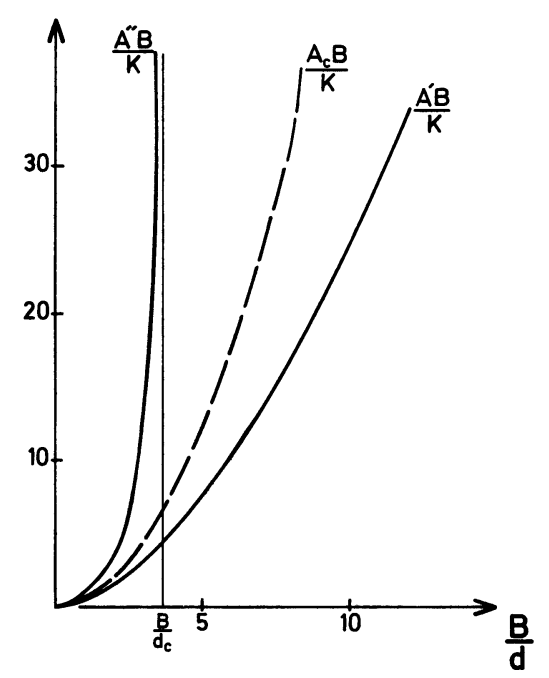

FIG. 3. - Reduced anchoring energy thresholds

$$
\frac{A_{\mathrm{c}} B}{K}, \frac{A^{\prime} B}{K}, \frac{A^{\prime \prime} B}{K} \text {. }
$$

3.2 At the higher threshold $\left(A \rightarrow A^{\prime \prime}\right)$ we have

$$
\varphi=\varphi_{0}\left(\frac{z}{d}\right)^{1 / 2} \frac{J_{1}(t)}{J_{1}\left(t_{0}\right)} .
$$

The ratio $\varphi / \varphi_{0}$ is positive and has the correct downward curvature for all physical values of $d$ whenever $d>d_{\mathrm{c}}=0.27 \mathrm{~B}$ (see Fig. $2 b$ ). The formula for the higher threshold is

$$
\frac{A^{\prime \prime} B}{K}=\left(\frac{B}{d}\right)^{3 / 2} \frac{J_{2}\left(2 \sqrt{\frac{B}{d}}\right)}{J_{1}\left(2 \sqrt{\frac{B}{d}}\right)}
$$

and the shape of $\varphi$ for large $z$ is

$$
\begin{aligned}
\varphi & =\varphi_{\infty}\left[1-\frac{B}{2 z}+O\left(\frac{B}{z}\right)^{2}\right], \quad z \gg B \\
\varphi_{\infty} & =\varphi_{0}\left(1+\frac{B}{2 d}\right) .
\end{aligned}
$$

This is represented on figure 3 . Note that $A^{\prime \prime}$ diverges when $d$ decreases down to $d_{\mathrm{c}}$. For $d<d_{\mathrm{c}}$, eq. (13) gives spatial oscillations which do not correspond to stable states. The final picture is as follows :

3.2.1 For $d>d_{\mathrm{c}}$ we have two transitions at $A=A^{\prime}$ and $A=A^{\prime \prime}$. In particular for $d \gg d_{\mathrm{c}}$ the relative interval of the oblique regime becomes very small

$$
\frac{A^{\prime \prime}-A^{\prime}}{A_{\mathrm{c}}}=\frac{1}{6}\left(\frac{B}{d}\right)^{3} .
$$

3.2.2 For $d<d_{\mathrm{c}}$ we have one transition at $A=A^{\prime}$. At all larger values of $A$ the conformation is oblique.

These theoretical transitions may have some bearing on recent observations by G. Ryschenkow and Kléman [10], using a slab of MBBA between two glass plates $\left(U \sim z^{-5}\right)$ coated with a certain carbon black. The anchoring is weak, it favors tangential (or nearly tangential) conditions at low temperatures, conical conditions at higher $T$, and finally reaches a homeotropic texture. The situation is complicated by various side effects [11] : but it may be connected to the local Frederiks transitions discussed here.

We must emphasize that our discussion on the order of the transition depends significantly on our 'assumption on the form of the surface energy $-\frac{I}{2} A \sin ^{2} \theta_{0}$. In many cases (and in particular for stereochemical fits at the molecular level) this energy is not expected to be a simple sinusoïdal function of $\theta_{0}:$ more often one would expect to have a sharp minimum at a certain $\theta_{0}$ value, and a flat plateau elsewhere. Situations of this set will require a separate discussion.

Finally, it must be mentioned that the geometry of figure $1 a$ is only one example among many : for instance it might be interesting to work with two competing planar geometries : if an uniaxial crystal (with axis $x$ parallel to the interface) is coated by a thin amorphous layer (thickness $d$ ) and the latter is grooved (or treated otherwise) to give a slight preference to the $y$ axis, a similar competition sets in and could be probed somewhat more easily. 


\section{References}

[1] De Gennes, P. G., C.R. Hebd. Séan. Acad. Sci. 271 (1970) 469.

[2] Richmond, P., White, L. R., Mol. Cryst. 27 (1974) 217.

[3] Smith, E. R., Ninham, B. W., Physica 66 (1973) 111.

[4] Dubois-Violette, E., De Gennes, P. G., to be published in J. Colloid Interface Sci.

[5] For a review on elastic constants, Frederiks transitions, and anchoring in nematics, see

De Gennes, The Physics of Liquid Crystals (Clarendon Press, Oxford) 1974.

[6] In practice we could work with a finite slab of thickness $2 D$ limited by two equivalent interfaces : imposing $\left.\frac{\mathrm{d} \theta}{\mathrm{d} z}\right|_{z=D}=0$ is probably correct in this case.
[7] Berremann, D. W., Mol. Cryst. Liq. Cryst. 21 (1973) 215.

[8] See Molecular Forces (edited by the Pontifical Academy, North Holland) 1967.

[9] For Bessel functions, see Handbook of Mathematical Functions (National Bureau of Standards).

[10] Ryschenkow, G., Thèse $3^{e}$ cycle, Orsay 1975.

Ryschenkow, G., Kléman, M., submitted to J. Chem. Phys.

[11] Very close to the nematic isotropic transition another tilt transition takes place. This could be explained if the temperature variations of $A$ and $U$ had similar, but not identical forms, so that the equations defining $A^{\prime}$, or $A^{\prime \prime}$ would have more than one root in temperature. 